\title{
CIAPIN1 and ABCA13 are markers of poor survival in metastatic ovarian serous carcinoma
}

\author{
Dag Andre Nymoen ${ }^{1}$, Arild Holth', Thea E Hetland Falkenthal' ${ }^{2}$, Claes G Tropé ${ }^{3,4}$ and Ben Davidson ${ }^{1,4^{*}}$
}

\begin{abstract}
Background: The objective of this study was to investigate the expression and clinical role of 14 genes previously shown to be associated with chemotherapy response and/or progression-free survival in a smaller series of ovarian serous carcinoma effusions.

Methods: Advanced-stage serous ovarian carcinoma effusions $(n=150)$ were analyzed for mRNA expression of AKR1C1, ABCA4, ABCA13, ABCB10, BIRC6, CASP9, CIAPIN1, FAS, MGMT, MUTYH, POLH, SRC, TBRKB and XPA using quantitative real-time $P C R$. mRNA expression was studied for association with clinicopathologic parameters, including chemotherapy response and survival.

Results: $A B C A 4$ mRNA expression was significantly related to better (complete) chemotherapy response at diagnosis in the entire cohort $(p=0.018)$, whereas higher POLH mRNA levels were significantly related to better chemoresponse at diagnosis in analysis to 58 patients with pre-chemotherapy effusions treated with standard chemotherapy (carboplatin + paclitaxel; $p=0.023)$. In univariate survival analysis for patients with pre-chemotherapy effusions $(n=77)$, CIAPIN1 mRNA expression was significantly related to shorter overall $(p=0.007)$ and progression-free $(p=0.038)$ survival, whereas ABCA13 mRNA expression was significantly related to shorter OS $(p=0.024)$. Higher CIAPIN1 mRNA expression was an independent marker of poor overall survival in Cox multivariate analysis $(p=0.044)$.
\end{abstract}

Conclusions: Our data identify $A B C A 4$ and $P O L H$ as markers of better chemotherapy response in metastatic serous carcinoma. CIAPIN1 and ABCA13 may be novel markers of poor outcome in pre-chemotherapy serous carcinoma effusions.

Keywords: Ovarian serous carcinoma, Effusions, Chemotherapy response, Survival

\section{Introduction}

Ovarian cancer, consisting predominantly of ovarian carcinoma (OC), is the most lethal gynecologic malignancy, constituting the 5th and 4th most common cause for cancer-related death in women in the U.S. and Norway, respectively $[1,2]$. Standard therapy consists of surgical cytoreduction followed by adjuvant chemotherapy using carboplatin and paclitaxel. While the majority of patients will respond to chemotherapy, the majority of tumors will recur within a median period of 18 months and become chemoresistant, resulting in 5-year survival of 30\% for advanced-stage disease [3]. Mechanisms by which

\footnotetext{
* Correspondence: bend@medisin.uio.no

'Department of Pathology, Oslo University Hospital, Norwegian Radium Hospital, Montebello, N-0310 Oslo, Norway

${ }^{4}$ University of Oslo, Faculty of Medicine, Institute of Clinical Medicine, N-0316 Oslo, Norway

Full list of author information is available at the end of the article
}

cancer cells acquire resistance to platinum agents include reduced cellular uptake, increased efflux, increased DNA repair and hypermethylation of $M L H 1$, part of the mismatch repair (MMR) system. Resistance to paclitaxel occurs through overexpression of multi-drug resistance protein 1 (MDR1), tubulin mutations and chromosomal instability [4]. Our current understanding of the molecular events that mediate chemoresistance in recurrent and metastatic OC is limited by the paucity of studies focusing on extra-ovarian disease.

OC progresses predominantly within the abdominal cavity and to a lesser degree by metastasis to the pleural space, frequently with the formation of malignant effusions. The serosal cavities are additionally a frequent site of disease recurrence [5]. We previously analyzed 32 serous OC effusions for the expression of 381 genes which have been reported to be associated with chemoresistance 
in OC using TaqMan arrays, and identified genes which were significantly associated with overall survival (OS), progression-free survival (PFS) and/or chemotherapy response [6]. In addition to its limited size, the latter study analyzed together primary diagnosis pre-chemotherapy effusions and effusions obtained post-chemotherapy, predominantly at disease recurrence. The objective of the present study was to analyze the expression of 14 genes found to be associated with chemoresponse and/or PFS in a larger cohort, consisting of 150 patients with serous OC effusions.

\section{Materials and methods}

\section{Patients and material}

Fresh non-fixed malignant peritoneal $(\mathrm{n}=125)$ and pleural $(n=25$; total $=150)$ effusions were obtained from 150 patients with FIGO stage III or IV serous ovarian, peritoneal or tubal carcinoma treated at the Department of Gynecologic Oncology at the Norwegian Radium Hospital in the period 1998-2008. Due to their closelylinked histogenesis and phenotype, all are referred to as OC henceforth. Informed consent was obtained according to national guidelines. The study was approved by the Regional Committee for Medical Research Ethics in Norway. Clinicopathologic data are detailed in Table 1.

Table 1 Clinicopathologic parameters of the study cohort (150 patients)

\begin{tabular}{|c|c|c|}
\hline \multicolumn{2}{|l|}{ Parameter } & \multirow{2}{*}{$\begin{array}{l}\text { Number of patients } \\
35-87 \text { years }(\text { mean }=62)\end{array}$} \\
\hline Age & & \\
\hline \multirow[t]{3}{*}{ Histological grade } & Low & 3 \\
\hline & High & 120 \\
\hline & $N A^{a}$ & 27 \\
\hline \multirow[t]{2}{*}{ FIGO stage } & III & 83 \\
\hline & IV & 67 \\
\hline \multirow[t]{3}{*}{ Residual disease } & $\leq 1 \mathrm{~cm}$ & 59 \\
\hline & $>1 \mathrm{~cm}$ & 63 \\
\hline & $N A^{b}$ & 28 \\
\hline CA 125 at diagnosis ${ }^{c}$ & & $11-43800$ years $($ mean $=3375)$ \\
\hline \multirow{5}{*}{$\begin{array}{l}\text { Chemoresponse after } \\
\text { primary treatment }^{d}\end{array}$} & $C R$ & 78 \\
\hline & PR & 32 \\
\hline & SD & 9 \\
\hline & PD & 21 \\
\hline & $\mathrm{NE}$ & 10 \\
\hline
\end{tabular}

${ }^{a} \mathrm{NA}=$ non available, including effusions from inoperable patients where biopsy was too small for grading or patients operated on in other hospitals, for which the primary tumor could not be accessed for assessment of grade.

${ }^{b} \mathrm{NA}=$ non available, including 4 patients who only received chemotherapy and 1 patient who received no therapy.

${ }^{c}$ CA 125 levels available for 104 patients.

${ }^{d} \mathrm{CR}=$ complete response, $\mathrm{PR}=$ partial response, $\mathrm{SD}=$ stable disease

$\mathrm{PD}=$ progressive disease, $\mathrm{NE}=$ disease response after chemotherapy could not be evaluated because of adverse effects, normalized CA-125 after primary surgery or missing CA-125 information and no residual tumor.
Eighty-six patients had primary surgery, 56 had secondary debulking and 4 patients only received chemotherapy. One patient received no therapy, and 3 patients referred at disease recurrence had no data available regarding therapy at diagnosis. Seventy-seven samples were obtained prior to chemotherapy administration, and 69 were obtained after chemotherapy, at interval debulking surgery or at recurrent disease. Chemotherapy status was unknown in 4 cases. The majority of patients $(n=138)$ received platinum-based therapy, of whom 101 received platinum + paclitaxel, 27 received single carboplatin and 10 received platinum combination. Of the remaining 12 patients, 10 received other chemotherapy and 1 received no therapy, whereas no information was available for 1 patient.

Effusions were submitted for routine diagnostic purposes and were processed immediately after tapping. Cell blocks were prepared using the Thrombin clot method. Diagnoses were established using morphology and immunohistochemistry (IHC). Effusion specimens were centrifuged immediately after tapping, and cell pellets were frozen at $-70^{\circ} \mathrm{C}$ in equal amounts of RPMI 1640 medium (GIBCO-Invitrogen, Carlsbad CA) containing 50\% fetal calf serum (PAA Laboratories $\mathrm{GmbH}$, Pasching, Austria) and 20\% dimethylsulfoxide (Merck KGaA, Darmstadt, Germany). Smears and H\&E-stained cell block sections were reviewed by a surgical pathologist experienced in cytopathology (BD). The tumor cell population was $>50 \%$ in all specimens.

\section{Quantitative real-time PCR (qRT-PCR)}

Effusions were centrifuged to obtain a cell pellet from which RNA was extracted using QIAsymphony (Qiagen, Hilden Germany). Details regarding reverse transcription, primer and probe design procedure and software, and efficiency testing were recently described [7]. The qRT-PCR reaction was run using the Perfecta qPCR ToughMix (Quanta Biosciences, Gaithersburg MD) and quantified on the Roche LightCycler 480 (Roche, Basel, Switzerland). Samples were analyzed in triplicate and average copy number was used for statistical analysis. Primer and probe sequences are detailed in Table 2.

The beta-glucuronidase (GUS), TATA box binding protein $(T B P)$ and mitochondrial ribosomal protein L19 (MRLP19) genes were used as reference genes following previous testing [7] applying established guidelines [8-10]. Primer and probe sequences were previously detailed [7].

IHC

Formalin-fixed paraffin-embedded sections from 109 of the 150 effusions analyzed using qRT-PCR were analyzed for Ciapin1 protein expression using the Dako EnVision ${ }^{\mathrm{m}}$ Flex + System (K8012; Dako, Glostrup, Denmark). Deparaffinization and unmasking of epitopes were carried out in 
Table 2 Primers and probes

\begin{tabular}{|c|c|}
\hline Gene & Sequence \\
\hline$A B C A 4$ & F-ACTTCTTCAAGCTCTTCCGTGTGC \\
\hline NM_000350.2 & R-TCAGATAATATTCCTCCCCAAGATCTCAG \\
\hline EXON 6-7 & P-CCACACTCCTAGACAGCCGTTCTCAAGGTATC \\
\hline$A B C A 13$ & F-CTGTGGAAGAATTGGCTCTGCA \\
\hline NM_152701.3 & R-CAGAATTACAAACAGGATACAAGGCCAG \\
\hline EXON 1-2 & P-CTCAGGAACCCGGTCCTITTCCTTGCTGAATTC \\
\hline BIRC6 & F-CACGTCCAGAACTCGGAGTG \\
\hline NM_016252.3 & R-AGGTAAATGTCTCCCGTCTGTTAGC \\
\hline EXON 4-5 & P-AGGCCGTTCTGTAGACAGATCACTGATGTATAGTG \\
\hline CASP9 & F-CAGACCAGTGGACATTGGTTCTG \\
\hline NM_001229.3 & R-CTCAGGATGTAAGCCAAATCTGCAT \\
\hline EKSON 3-4 & P-TGGTGATGTCGGTGCTCTTGAGAGTTTGAG \\
\hline CIAPIN1 & F-CACCAAGAAGTCTTCTCCTTCAGTG \\
\hline NM_020313.2 & R-GCTGAGAGGGTCCACAGCT \\
\hline EXON 5-6 & P-ACCTGCTGTGGACCCTGCTGCTG \\
\hline MGMT & F-CATCCCGTTTCCAGCAAGAG \\
\hline NM_002412.3 & R-GGTAAGAAATCACTTCTCCGAATTTCA \\
\hline EXON 3-4 & P-TTCACCAGACAGGTGTTATGGAAGCTGCTGAAG \\
\hline TPRKB & F-GGGAGCTCTTCCGAGACG \\
\hline NM_016058.2 & R-GTCCAGCTGATGTGTTAACTGCAT \\
\hline EXON 1-2 & P-TGGGGGCCGGATGTAGAATCCTGCTTA \\
\hline$X P A$ & F-CACAATGGGGTGATATGAAACTCTACT \\
\hline NM_000380.3 & R-CCTTTGCTTCTTCTAATGCTTCTTGACT \\
\hline EXON 4-5 & P-AAGTTACAGATTGTGAAGAGGTCTCTTGAAGTTTGGG \\
\hline POLH & F-TATGTCCAGATCTTCTACTGGCACAAGT \\
\hline NM_006502.2 & R-AAAACGAGACATTATCTCCATCACTTCA \\
\hline EXON 3-4 & P-CGTGGGAAAGCTAACCTCACCAAGTACCGG \\
\hline FAS & F-GATTGCTCAACAACCATGCTG \\
\hline NM_000043.4 & R-GGGCATTAACACTTTTGGACGAT \\
\hline EXON 1-2 & P-TGGACCCTCCTACCTCTGGTTCTTACGTCTGTTGCTAG \\
\hline$S R C$ & F-CCCCTGCCTTCTACCAGGAC \\
\hline NM_005417.3 & R-CGGCGGGCTCCAGGCT \\
\hline EXON 3-4 & P-TGGGTAGCAACAAGAGCAAGCCCAAGGATG \\
\hline AKR1C1 & F-CATCAGACAGAACGTGCAGGTG \\
\hline NM_001353.5 & R-CAAAAATATCAAGGGTCAAATATCGC \\
\hline EXON 7-8 & P-CCAGTTGACTTCAGAGGAGATGAAAGCCATAGATG \\
\hline MUTYH & F-TCCACCGCCATGAAAAAGGT \\
\hline NM_001048171.1 & R-GACCTITTGGAACCCATACAGGTC \\
\hline EXON 14-15 & P-TCCGTGTGTATCAGGGCCAACAGCCA \\
\hline$A B C B 10$ & F-ACTCTCTTCCTTCCTAATGTATGCTTTCTG \\
\hline NM_012089.2 & R-CACCCAGTCCTTTCATCAGCT \\
\hline EXON 6-4 & P-TGGAATAAGCATTGGAGGTCTGAGCTCTTTCTACTC \\
\hline
\end{tabular}

$\mathrm{F}=$ Forward primer; $\mathrm{R}=$ Reverse primer; $\mathrm{P}=$ Probe. Probes are labeled with $5^{\prime}$ Fam and 3'non fluorescent quencher. a PT-Link (Dako) using an EnVision ${ }^{\mathrm{Tm}}$ Flex target retrieval solution at a low pH (Tris/EDTA pH 6). Sections were incubated with a $0.3 \%$ hydrogen peroxide $\left(\mathrm{H}_{2} \mathrm{O}_{2}\right)$ solution for 5 min to block endogeneous tissue peroxidase activity. Sections were incubated with a rabbit polyclonal Ciapin1 antibody (NBP1-89097, Novus Biologicals, Littleton, CO; 1:100 dilution), and then treated with EnVision ${ }^{\mathrm{Tm}}$ Flex + mouse linker (15 min) and EnVision ${ }^{\mathrm{Tm}}$ Flex/HRP enzyme (30 min). Sections were stained for $10 \mathrm{~min}$ with 3'3-diaminobenzidine tetrahydrochloride (DAB), counterstained with hematoxylin, dehydrated and mounted in RichardAllan Scientific Cyto seal XYL (Thermo Fisher Scientific, Waltham, MA). Positive and negative controls consisted of bladder carcinoma and sections stained with rabbit serum, respectively.

\section{Interpretation}

Staining was considered positive when localized to the cytoplasm or nucleus. Staining extent was scored on a scale of $0-4$, corresponding to staining of $0 \%, 1-5 \%, 6-$ $25 \%, 26-75 \%$ and $76-100 \%$ of cells. No specimen contained less than 100 tumor cells. Slides were scored by a surgical pathologist experienced in effusion cytology (BD).

\section{Statistical analysis}

Statistical analysis was performed applying the SPSS-PC package (Version 18, Chicago IL). Probability of $<0.05$ was considered statistically significant. Analysis of the association between mRNA levels of the 14 studied genes, as well as Ciapin 1 protein expression, and clinicopathologic parameters was performed using the Mann-Whitney $U$ test. For this analysis, as well as for survival analysis, clinicopathologic parameters were grouped as follows: Age: $\leq 60$ vs. $>60$ years; effusion site: peritoneal vs. pleural; FIGO stage: III vs. IV; chemotherapy status: pre- vs. postchemotherapy specimens; Residual disease (RD): $\leq 1 \mathrm{~cm}$ vs. $>1 \mathrm{~cm}$; chemotherapy response: complete vs. partial response/stable disease/progression; primary (intrinsic) chemoresistance: PFS $\leq 6$ months vs. $>6$ months. Histological grade was not used for statistical analyses due to the small number of low-grade tumors. The paired-sample T-test was used to analyze the association between mRNA expression levels of the studied genes and CA 125 levels.

PFS and OS were calculated from the date of the last chemotherapy treatment/diagnosis to the date of recurrence/death or last follow-up, respectively. Univariate survival analyses of PFS and OS were executed using the Kaplan-Meier method and log-rank test. Platinum resistance was defined as PFS $\leq 6$ months according to guidelines published by the Gynecologic Oncology Group (GOG) [11] and progressive disease or recurrence was evaluated by the RECIST criteria [12]. Multivariate analysis was performed using the Cox Regression Model (Enter function). 


\section{Results}

Chemoresistance-associated genes are significantly related to clinicopathologic parameters

Analysis of mRNA levels for the 14 studied genes in the 150 effusions identified several significant associations with anatomic site, patient age and residual disease volume (Table 3). No significant associations were found with chemotherapy status (pre- vs. post-chemotherapy specimens) or with FIGO stage ( $\mathrm{p}>0.05)$.

Our previous research has demonstrated that pre- and post-chemotherapy effusions differ considerably with respect to expression of cancer-associated molecules and their clinical relevance [5]. Despite the absence of significant differences in expression of the 14 studied genes among pre- and post-chemotherapy effusions, we therefore analyzed them separately.

Significant associations for pre-chemotherapy cases are shown in Table 4. In analysis of post-chemotherapy effusions, only age was significantly associated with gene expression, with associations identified between older age and lower $A B C A 4(\mathrm{p}=0.02)$ and higher BIRC6 ( $\mathrm{p}=0.03)$, $A B C A 13(\mathrm{p}=0.008)$ and $F A S(\mathrm{p}=0.018)$ levels.

Ciapin1 protein was expressed in the cytoplasm of OC cells in 92/109 effusions, of which 28 had score =1, 25 score $=2,24$ score $=3$ and 15 score $=4$ (Figure 1). Nuclear localization was observed in $<10$ cases and was not scored (Figure 1). Expression in reactive mesothelial cells was observed in 2 cases, while staining was tumorspecific in the remaining effusions.

$A B C A 4$ and POLH mRNA expression is related to complete chemotherapy response at diagnosis

In analysis of the 144 patients which had data regarding chemotherapy response at diagnosis, higher $A B C A 4$ mRNA expression was significantly related to better

Table 3 Significant associations between gene expression and clinicopathologic parameters in the entire study cohort (150 patients)

\begin{tabular}{|c|c|c|c|}
\hline Parameter & Gene & Overexpression & P-value \\
\hline \multirow[t]{2}{*}{ Anatomic site } & $A B C A 13$ & Peritoneum & $p=0.03$ \\
\hline & FAS & Pleura & $p=0.041$ \\
\hline \multirow[t]{4}{*}{ Age } & $A B C B 10$ & Age $>60$ years & $p=0.006$ \\
\hline & BIRC6 & Age $>60$ years & $p=0.004$ \\
\hline & $A B C A 13$ & Age $>60$ years & $p=0.011$ \\
\hline & CASPO & Age $>60$ years & $p=0.049$ \\
\hline \multirow[t]{4}{*}{ Residual disease } & $X P A$ & $\leq 1 \mathrm{~cm}$ & $p=0.046$ \\
\hline & MGMT & $\leq 1 \mathrm{~cm}$ & $p=0.04$ \\
\hline & TBRKB & $\leq 1 \mathrm{~cm}$ & $p=0.002$ \\
\hline & $A B C B 10$ & $\leq 1 \mathrm{~cm}$ & $p=0.011$ \\
\hline \multirow[t]{2}{*}{ CA 125 levels at diagnosis } & $A B C A 4$ & Positive association & $p=0.011$ \\
\hline & $A B C B 10$ & Negative association & $p=0.01$ \\
\hline
\end{tabular}

Table 4 Significant associations between gene expression in pre-chemotherapy effusions and clinicopathologic parameters (77 patients)

\begin{tabular}{llll}
\hline Parameter & Gene & Overexpression & P-value \\
\hline Anatomic site & FAS & Pleura & $\mathrm{p}=0.034$ \\
Age & $A B C B 10$ & Age $>60$ years & $\mathrm{p}=0.005$ \\
FIGO stage & MUYTH $\quad$ II & $\mathrm{p}=0.03$ \\
& $A B C A 4 \quad$ III & $\mathrm{p}=0.011$ \\
Residual disease & $M G M T \quad \leq 1 \mathrm{~cm}$ & $\mathrm{p}=0.007$ \\
& TBRKB $\leq 1 \mathrm{~cm}$ & $\mathrm{p}=0.003$ \\
& $A B C B 10 \leq 1 \mathrm{~cm}$ & $\mathrm{p}=0.014$ \\
CA 125 levels at diagnosis & $A B C A 4$ & Positive association & $\mathrm{p}=0.003$ \\
& $A B C B 10$ & Negative association & $\mathrm{p}=0.042$ \\
\hline
\end{tabular}

(complete) chemoresponse at diagnosis $(\mathrm{p}=0.018)$. In evaluation of the relationship between gene expression and primary chemoresistance in the entire cohort, no significant associations were found $(\mathrm{p}>0.05)$. However, trends for association between high ABCA13 ( $\mathrm{p}=0.059)$ and low $F A S(\mathrm{p}=0.053)$ mRNA levels with primary chemoresistance were observed.

In analysis of the 73 patients with pre-chemotherapy effusions which had data regarding chemotherapy response at diagnosis, higher CIAPIN1 mRNA expression was associated with a trend for worse (non-complete) chemoresponse at diagnosis $(\mathrm{p}=0.052)$. A trend was additionally found between low FAS mRNA levels and primary chemoresistance $(\mathrm{p}=0.056)$.

Limiting the analysis to 58 patients with prechemotherapy effusions treated with standard chemotherapy (carboplatin + paclitaxel) identified POLH mRNA as marker better chemoresponse at diagnosis $(\mathrm{p}=0.023)$. Ciapin 1 protein expression was unrelated to chemotherapy response.

CA 125 level at diagnosis, available for 59 patients with pre-chemotherapy effusions, was not significantly related to chemoresponse at diagnosis $(p=0.207)$. HE4, which is currently measured in all OC patients at our hospital, was not measured in the period the effusions studied in this series were collected (1998-2008), and therefore cannot be assessed for performance in this study.

None of the studied genes was related to chemoreponse at diagnosis or to primary chemoresistance in the group of patients with post-chemotherapy effusions $(p>0.05)$.

\section{CIAPIN1 and $A B C A 13$ are prognostic markers in pre-chemotherapy serous $O C$ effusions}

The follow-up period for the 150 patients ranged from 1 to 156 months ( mean $=33$ months, median $=24$ months ). PFS, available for 149 patients, ranged from 0 to 116 months (mean $=9$ months, median $=5$ months). At the last follow-up, 1 patient was alive with no evidence 


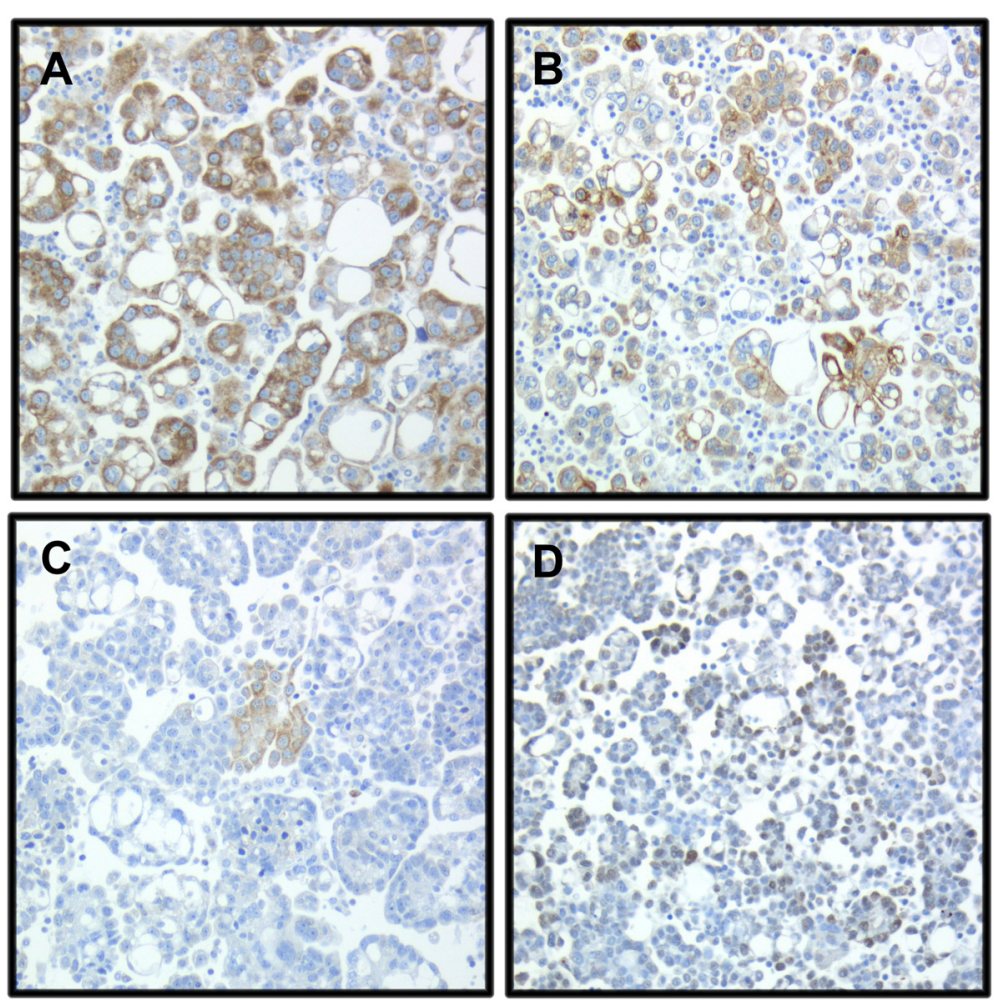

Figure 1 Ciapin1 protein expression by immunohistochemistry. (A-B) Diffuse Ciapin 1 expression (>75\% of tumor cells) in the cytoplasm of OC cells in 2 effusions; (C) Focal expression $(<5 \%)$ in another specimen; (D) Nuclear localization of Ciapin1, an unusual finding in this series.

of disease, 6 were alive with disease and 142 were dead of disease. One patient died of unrelated causes.

In survival analysis for all patients, none of the studied genes was significantly related to OS or PFS. The same findings were observed for the patient group with postchemotherapy effusions. However, in univariate survival analysis for patients with pre-chemotherapy effusions $(\mathrm{n}=77)$, CIAPIN1 mRNA expression was significantly related to shorter OS $(\mathrm{p}=0.007$; Figure $2-\mathrm{A})$ and PFS $(\mathrm{p}=0.038$; Figure 2-B). In addition, $A B C A 13 \mathrm{mRNA}$ expression was significantly related to shorter OS $(\mathrm{p}=0.024$; Figure 2-C), though not with PFS ( $\mathrm{p}=0.192) . A B C A 4$ and $P O L H$ expression was not significantly related to survival, and the same was true for Ciapin1 protein expression.

Among the clinical parameters, older age was significantly related to shorter OS $(\mathrm{p}=0.044)$ and was associated with a trend for poor PFS ( $p=0.055)$. RD volume $>1 \mathrm{~cm}$ was significantly related to poor PFS $(\mathrm{p}=0.031)$, with nonsignificant association with OS $(\mathrm{p}=0.196)$. CA 125 level at diagnosis in patients with pre-chemotherapy effusions were unrelated to PFS ( $p=0.185)$ or to OS ( $p=0.413$ ) in this patient group.

The parameters entered into Cox analysis of OS and PFS were all those with $\mathrm{p}<0.2$, i.e. CIAPIN1 and $A B C A 13$ mRNA expression, RD volume and age for both endpoints. In Cox multivariate analysis of OS, higher
CIAPIN1 mRNA expression was the only independent marker of poor outcome $(\mathrm{p}=0.044)$. In Cox multivariate analysis of PFS, larger RD was the sole independent marker $(\mathrm{p}=0.041)$.

In univariate survival analysis for patients with prechemotherapy effusions who received combination chemotherapy with carboplatin and paclitaxel $(n=58)$, ABCA13 mRNA expression $(\mathrm{p}=0.006)$ and older age $(\mathrm{p}=0.026)$ were significantly related to shorter OS. Non-significant associations with shorter OS were observed for CIAPIN1 mRNA expression $(\mathrm{p}=0.146)$ and RD volume $(\mathrm{p}=0.141)$. In Cox multivariate analysis, none of these markers was an independent prognosticator.

\section{Discussion}

Intrinsic and acquired drug resistance remains a major therapeutic obstacle in OC [3], and our understanding of the cellular mediators of this phenomenon in metastatic OC cells is limited by the paucity of large series of such specimens. In the present study we assessed the predictive and prognostic role of 14 genes identified as potentially clinically relevant in an earlier study [6]. Our data only partially confirm the initial report. Inconsistent results from different mRNA expression studies are not infrequent and different methodology is the major cause of these discrepancies [13]. In our case the initial study 


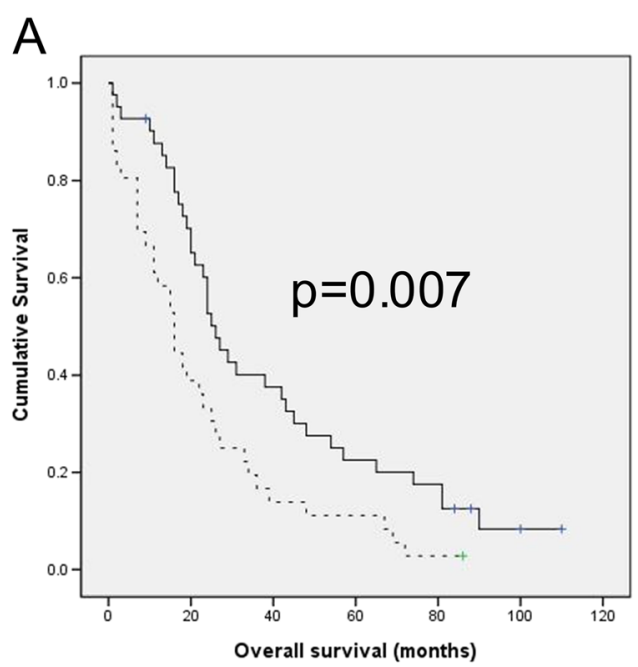

B

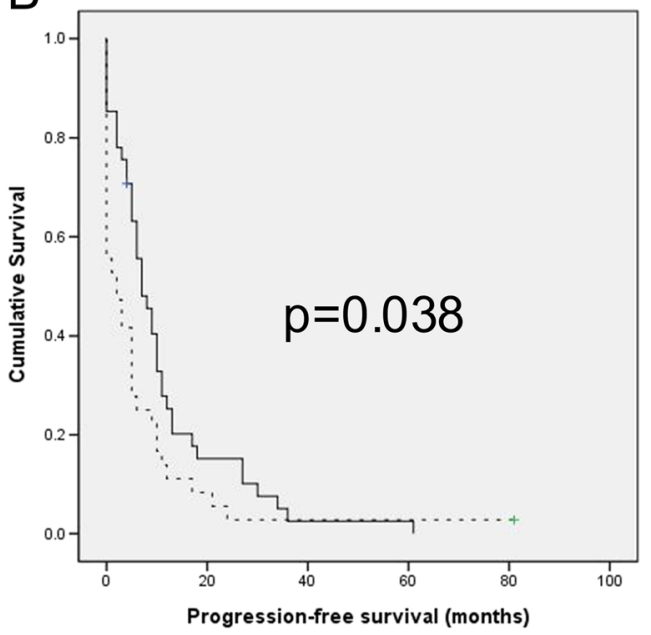

C

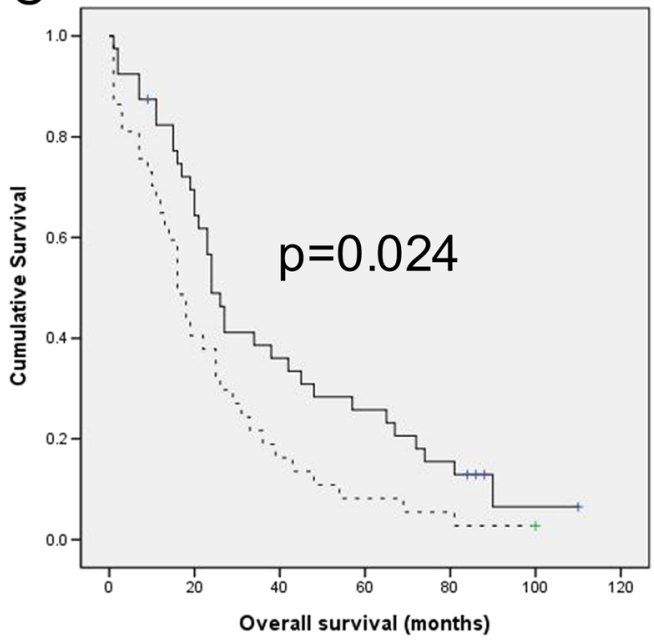

Figure 2 CIAPIN1 and ABCA13 mRNA expression is associated with shorter survival in serous ovarian carcinoma. $\mathbf{A}$ : Kaplan-Meier survival curve showing the association between CIAPIN1 mRNA expression in effusions and overall survival (OS) for patients with pre-chemotherapy primary diagnosis effusions $(n=77)$. Patients with effusions with higher-than-median expression ( $n=36$, dashed line) had a mean OS of 22 months vs. 40 months for patients whose effusions showed lower-than-median expression ( $n=41$, solid line; $p=0.007$ ). B: Kaplan-Meier survival curve showing the association between CIAPIN1 mRNA expression in effusions and progression-free survival (PFS) for patients with pre-chemotherapy primary diagnosis effusions $(n=77)$. Patients with effusions with higher-than-median expression ( $n=36$, dashed line) had a mean PFS of 7 months vs. 11 months for patients whose effusions showed lower-than-median expression ( $n=41$, solid line; $p=0.038$ ).

C: Kaplan-Meier survival curve showing the association between ABCA13 mRNA expression in effusions and OS for patients with pre-chemotherapy primary diagnosis effusions $(n=77)$. Patients with effusions with higher-than-median expression ( $n=36$, dashed line) had a mean OS of 24 months vs. 39 months for patients whose effusions showed lower-than-median expression ( $n=41$, solid line; $p=0.024$ ).

used a Taqman array assay and $18 S$ for normalization vs. our in-house assay in which primers were designed by our group and the average of 3 reference genes previously shown to be optimal for OC analysis was used for normalization. The number of samples also differed considerably, being 32 in the previous study vs. 150 in the present one. Some findings were nevertheless reproduced, as CIAPIN1 and ABCA13 expression had prognostic value in pre-chemotherapy patients and $A B C A 4$ and $P O L H$ were predictive for complete chemotherapy response at diagnosis. Additional correlation with clinopathologic parameters was found for anatomic site (ABCA13 and FAS), patient age (ABCB10, BIRC6, $A B C A 13$ and $C A S P 9)$ and residual disease volume (XPA, $M G M T, T B R K B$ and $A B C B 10)$. Though no association was observed between gene levels and previous chemotherapy or FIGO stage in analysis of the entire cohort, FIGO stage was significantly associated with MUTYH and $A B C A 4$ expression in pre-chemotherapy disease.

Three members of the ATP binding cassette (ABC) family were assessed on the present study. These transmembrane proteins serve as efflux pumps and members like MDR1 play a major role in multidrug chemoresistance $[14,15]$. The role of ABCA4 in cancer is largely unknown to date, whereas $\mathrm{ABCB} 10$ was previously shown to be expressed in drug-resistant HT-29 colon carcinoma cells [16]. Higher $A B C A 13$ levels by qPCR have been associated with better response to neoadjuvant chemotherapy in breast cancer [17] and with longer disease-free survival following chemotherapy in colorectal cancer [18], and this gene was previously reported to be underexpressed in renal cell carcinoma compared to normal tissue [19]. Our data associate $A B C A 4$ with better chemotherapy response at diagnosis. However, the 
marginal association between higher $A B C A 13$ levels and primary chemoresistance and the significant association with poor OS suggest that this molecule may have a different role in serous OC.

The present study identified CIAPIN1 as an independent prognostic marker of poor outcome for patients with pre-chemotherapy effusions. Cytokine induced apoptosis inhibitor 1 (CIAPIN1) is an anti-apoptotic protein exerting its function through post-translational modifications of cell cycle proteins including p27, cyclins D1 and E and the cyclin-dependent kinases CDK2 and CDK4. It additionally cooperates with other proteins, such as thioredoxin-like protein 2 (TXNL2), to inhibit apoptosis via the mitochondrial (intrinsic) pathway. Ciapin1 additionally regulates cell differentiation, organ development and cellular stress response [20]. Nuclear expression of CIAPIN1 was reported to be associated with poor prognosis in a study of 108 primary OC [21]. In our series, this protein was predominantly localized to the cytoplasm, nuclear expression being too infrequent to be considered of clinical relevance. This emphasizes the differences between primary OC and effusion specimens, but may also reflect the fact that the study by Cai et al. included both serous and non-serous tumors, whereas our series consisted uniformly of the former.

$P O L H$, encoding Polymerase $\eta$, was associated with complete response to primary chemotherapy in the patient group with pre-chemotherapy effusions treated with standard chemotherapy (carboplatin + paclitaxel). POLH, a.k.a. XPV and RAD30A, is member of the Yfamily of DNA polymerases. Mutation in this protein is the basis for the Variable type of Xeroderma Pigmentosum, a genetic disease with impaired DNA repair associated with skin cancer [22]. Polymerase $\eta$ was further reported to mediate resistance to cisplatin by extending primers after DNA cross-linking induced by the drug [23]. Our data do not support this role and rather constitute the first report attributing a potential beneficial clinical role to this molecule in OC.

The remaining genes analyzed in this study were not informative for chemotherapy response or survival. Protein expression of FAS, activator of the extrinsic apoptosis pathway, by flow cytometry in $\mathrm{OC}$ effusions was previously reported to be a marker for aggressive clinical behavior by our group [24], but its mRNA was significantly related only to anatomic localization (pleura vs. peritoneum) in the present study. Similarly, protein expression of the DNA repair gene XPA was previously found to be associated with better prognosis by our group [25], whereas XPA mRNA expression was unrelated to survival in the present study. These discrepancies likely reflect different methodology, as well as the fact that protein and mRNA expression do not fully overlap in many cases.
The inhibitor of apoptosis (IAP) family consists of 8 proteins which are key regulators of apoptosis. IAP members are overexpressed in many cancers and are under considerable attention as therapeutic targets $[26,27]$. Protein expression of the IAP member Apollon (a.k.a. Baculoviral IAP Repeat Containing 6; BIRC6) in primary $\mathrm{OC}$ was recently reported to be related to poor prognosis [28], an association which was not reproduced in our analysis of BIRC6 mRNA levels in effusions. Similarly, SRC, modulator of response to paclitaxel and a potential molecular target in OC [29], was not informative of therapy response or survival in our series.

TPRKB, a.k.a. TP53RK, binds and activates p53 by phosphorylation. The gene is located in the 20q13.12-13 region which is amplified in OC and was reported to be associated with primary chemoresistance [30]. TP53RK silencing activates caspases 3 and 7 and sensitizes OC cells to paclitaxel [31].

MUTYH is a DNA glycosylase that repairs oxidative damage by replacing an incorrectly placed A with $\mathrm{C}$, thereby preventing mutations. However, it appears to have additional roles, primarily as pro- and antiapoptotic marker, and is localized to both the mitochondria and the nucleus. MUTYH is implicated in cancer, inflammation and Parkinson disease. Mutations in MUTYH predispose individuals to colorectal cancer [32], as well as increased risk of developing OC [33].

O-6-methylguanine-DNA methyltransferase (MGMT), an enzyme regulated primarily through promoter methylation, repairs DNA damage caused by alkylating lesions and thus rescues tumor cells from apoptosis [34]. Higher MGMT activity was reported to be associated with cisplatin resistance in OC [35].

AKR1C1 is family member of the Aldo-keto reductases, and have been reported to enhance progesterone metabolism in ovarian endometriosis [36].

A relevant question concerning the results of the present study would be how other biomarkers perform in this cohort with respect to predicting chemotherapy response or as prognostic factors. We recently published a retrospective analysis of 143 serous OC effusions in which we assessed the clinical role of 41 previously studied proteins. In this series, several proteins that were significantly related to chemotherapy response and/or survival (the latter in pre-chemotherapy effusions) were identified, including survivin, fatty acid synthase (FAS), p21-activated kinase-1 (PAK1), osteopontin, peroxisome proliferator-activated receptor- $\gamma$ (PPAR $\gamma$ ), S100A4, Bcl$\mathrm{XL}$, high-mobility group protein A2 (HMGA2), NAC-1, signal transducer and activator of transcription $5 \mathrm{~B}$ (STAT5B) and heat shock protein 90 (HSP90) [37]. Analysis of the clinical role of these markers in the prechemotherapy specimens analyzed in the present study identified only nuclear survivin expression as significantly 
related to chemotherapy response (favorable; $\mathrm{p}=0.017$ ) or OS (favorable; $\mathrm{p}=0.013$ ). It should nevertheless be commented that the number of overlapping specimens was 47 or less for these markers. We are currently performing a similar multivariate analysis for biomarkers studied at the mRNA level, which may complement the current study in identifying clinically relevant targets in OC effusions.

In conclusion, analysis of 14 chemoresistance-associated genes in a large series of serous $\mathrm{OC}$ effusions identified CIAPIN1, ABCA4, ABCA13 and POLH as potential markers of survival or chemotherapy response in this setting, findings which merit in our opinion further research. Multiple associations were additionally identified between the studied genes and various clinicopathologic parameters. While some of the latter may represent errors of multiple testing, the stronger associations $(p<0.005)$ may represent true findings. Sorting the clinically relevant from the non-relevant molecular markers in recurrent and/or metastatic OC may aid in selection of therapeutic targets in this cancer.

\section{Competing interest}

The authors declare that they have no competing interests.

\section{Authors' contributions}

DAN designed the GPCR assay, performed the GPCR analysis, participated in performing the statistical analysis, and wrote the manuscript; AH performed the immunohistochemistry analysis; TEHF and CGT provided the clinical data for the study cohort; BD designed the study, performed the statistical analysis and supervised the writing of this manuscript. All authors read and approved the final manuscript.

\section{Acknowledgment}

This work was supported by Inger and John Fredriksen Foundation for Ovarian Cancer Research.

\section{Author details}

'Department of Pathology, Oslo University Hospital, Norwegian Radium Hospital, Montebello, N-0310 Oslo, Norway. ${ }^{2}$ Department of Oncology, Oslo University Hospital, Norwegian Radium Hospital, N-0310 Oslo, Norway. ${ }^{3}$ Department of Gynecologic Oncology, Oslo University Hospital, Norwegian Radium Hospital, N-0310 Oslo, Norway. ${ }^{4}$ University of Oslo, Faculty of Medicine, Institute of Clinical Medicine, N-0316 Oslo, Norway.

Received: 14 November 2014 Accepted: 9 February 2015

Published online: 18 February 2015

\section{References}

1. Siegel R, Ma J, Zou Z, Jemal A. Cancer statistics, 2014. CA Cancer J Clin. 2014;64:9-29.

2. Cancer Registry of Norway. Cancer in Norway 2010 - Cancer incidence, mortality, survival and prevalence in Norway. Oslo: Cancer Registry of Norway; 2012

3. Colombo PE, Fabbro M, Theillet C, Bibeau F, Rouanet P, Ray-Coquard I. Sensitivity and resistance to treatment in the primary management of epithelial ovarian cancer. Crit Rev Oncol Hematol. 2014;89:207-16.

4. Holohan C, Van Schaeybroeck S, Longley DB, Johnston PG. Cancer drug resistance: an evolving paradigm. Nat Rev Cancer. 2013;13:714-26.

5. Davidson B. Ovarian and Primary Peritoneal Carcinoma. In: Davidson B, Firat P, Michael CM, editors. Serous Effusions- Etiology, Diagnosis, Prognosis and Therapy. London: Springer; 2011. p. 167-204.

6. Gillet JP, Wang J, Calcagno AM, Green LJ, Varma S, Bunkholt Elstrand M, et al. Clinical relevance of multidrug resistance gene expression in ovarian serous carcinoma effusions. Mol Pharm. 2011;8:2080-8.
7. Hetland TE, Nymoen DA, Emilsen E, Kærn J, Tropé CG, Flørenes VA, et al. MGST1 expression in serous ovarian carcinoma differs at various anatomic sites, but is unrelated to chemoresistance or survival. Gynecol Oncol. 2012;126:460-5.

8. Bustin SA, Benes V, Garson JA, Hellemans J, Huggett J, Kubista M, et al. The MIQE Guidelines: Minimum Information for Publication of Quantitative RealTime PCR Experiments. Clin Chem. 2009;55:611-22.

9. Andersen $C L$, Jensen $J L$, Orntoft $T$. Normalization of real-time quantitative reverse transcription-PCR data: a model-based variance estimation approach to identify genes suited for normalization, applied to bladder and colon cancer data sets. Cancer Res. 2004;64:5245-50.

10. Vandesompele J, De Preter K, Pattyn F, Poppe B, Van Roy N, De Paepe A, et al. Accurate normalization of real-time quantitative RT-PCR data by geometric averaging of multiple internal control genes. Genome Biol 2002;3:RESEARCH0034.

11. Thigpen JT, Blessing JA, Ball H, Hummel SJ, Barrett RJ. Phase II trial of paclitaxel in patients with progressive ovarian carcinoma after platinumbased chemotherapy: a Gynecologic Oncology Group study. J Clin Oncol. 1994;12:1748-53.

12. Therasse P, Arbuck SG, Eisenhauer EA, Wanders J, Kaplan RS, Rubinstein L, et al. New quidelines to evaluate the response to treatment in solid tumors. European Organization for Research and Treatment of Cancer, National Cancer Institute of the United States, National Cancer Institute of Canada. J Natl Cancer Inst. 2000;92:205-16.

13. Fekete T, Rásó E, Pete I, Tegze B, Liko I, Munkácsy G, et al. Meta-analysis of gene expression profiles associated with histological classification and survival in 829 ovarian cancer samples. Int J Cancer. 2012;131:95-105.

14. Gottesman MM, Ling V. The molecular basis of multidrug resistance in cancer: the early years of P-glycoprotein research. FEBS Lett. 2006;580:998-1009.

15. Sharom FJ. ABC multidrug transporters: structure, function and role in chemoresistance. Pharmacogenomics. 2008;9:105-27.

16. Yasui K, Mihara S, Zhao C, Okamoto H, Saito-Ohara F, Tomida A, et al. Alteration in copy numbers of genes as a mechanism for acquired drug resistance. Cancer Res. 2004;64:1403-10.

17. Hlaváč V, Brynychová V, Václavíková R, Ehrlichová M, Vrána D, Pecha V, et al. The expression profile of ATP-binding cassette transporter genes in breast carcinoma. Pharmacogenomics. 2013;14:515-29.

18. Hlavata I, Mohelnikova-Duchonova B, Vaclavikova R, Liska V, Pitule P, Novak $P$, et al. The role of $A B C$ transporters in progression and clinical outcome of colorectal cancer. Mutagenesis. 2012;27:187-96.

19. Arai E, Sakamoto H, Ichikawa H, Totsuka H, Chiku S, Gotoh M, et al. Multilayer-omics analysis of renal cell carcinoma, including the whole exome, methylome and transcriptome. Int J Cancer. 2014;135:1330-42.

20. Li X, Wu K, Fan D. CIAPIN1 as a therapeutic target in cancer. Expert Opin Ther Targets. 2010;14:603-10.

21. Cai X, Wang J, Xin X. CIAPIN1 nuclear accumulation predicts poor clinical outcome in epithelial ovarian cancer. World J Surg Oncol. 2012;10:112.

22. Sale JE, Lehmann AR, Woodgate R. Y-family DNA polymerases and their role in tolerance of cellular DNA damage. Nat Rev Mol Cell Biol. 2012;13:141-52.

23. Zhao Y, Biertümpfel C, Gregory MT, Hua YJ, Hanaoka F, Yang W. Structural basis of human DNA polymerase n-mediated chemoresistance to cisplatin. Proc Natl Acad Sci U S A. 2012;109:7269-74.

24. Dong HP, Kleinberg L, Silins I, Flørenes VA, Trope' CG, Risberg B, et al. Death receptor expression is associated with poor response to chemotherapy and shorter survival in metastatic ovarian carcinoma. Cancer. 2008;112:84-93.

25. Stevens EV, Raffeld M, Espina V, Kristensen GB, Trope' CG, Kohn EC, et al. Expression of Xeroderma Pigmentosum A protein predicts improved outcome in metastatic ovarian carcinoma. Cancer. 2005;103:2313-9.

26. Fulda S, Vucic D. Targeting IAP proteins for therapeutic intervention in cancer. Nat Rev Drug Discov. 2012;11:109-24.

27. Infante JR, Dees EC, Olszanski AJ, Dhuria SV, Sen S, Cameron S, et al. Phase I Dose-Escalation Study of LCL161, an Oral Inhibitor of Apoptosis Proteins Inhibitor, in Patients With Advanced Solid Tumors. J Clin Oncol. 2014;32:3103-10

28. Wang L, Chen YJ, Hou J, Wang YY, Tang WQ, Shen XZ, et al. Expression and clinical significance of BIRC6 in human epithelial ovarian cancer. Tumour Biol. 2014;35:4891-6.

29. Le XF, Bast Jr RC. Src family kinases and paclitaxel sensitivity. Cancer Biol Ther. 2011;12:260-9.

30. Etemadmoghadam D, DeFazio A, Beroukhim R, Mermel C, George J, Getz G, et al. AOCS Study Group: integrated genome-wide DNA copy number and 
expression analysis identifies distinct mechanisms of primary chemoresistance in ovarian carcinomas. Clin Cancer Res. 2009;15:1417-27.

31. Peterson D, Lee J, Lei XC, Forrest WF, Davis DP, Jackson PK, et al. A chemosensitization screen identifies TP53RK, a kinase that restrains apoptosis after mitotic stress. Cancer Res. 2010;70:6325-35.

32. Markkanen E, Dorn J, Hübscher U. MUTYH DNA glycosylase: the rationale for removing undamaged bases from the DNA. Front Genet. 2013;4:18.

33. Vogt S, Jones N, Christian D, Engel C, Nielsen M, Kaufmann A, et al. Expanded extracolonic tumor spectrum in MUTYH-associated polyposis. Gastroenterology. 2009;137:1976-85.

34. Cankovic M, Nikiforova MN, Snuderl M, Adesina AM, Lindeman N, Wen PY, et al. The Role of MGMT Testing in Clinical Practice A Report of the Association for Molecular Pathology. J Mol Diagn. 2013;15:539-55.

35. Chen SS, Citron M, Spiegel G, Yarosh D. O6-methylguanine-DNA methyltransferase in ovarian malignancy and its correlation with postoperative response to chemotherapy. Gynecol Oncol. 1994;52:172-4

36. Hevir N, Vouk K, Sinkovec J, Ribič-Pucelj M, Rižner TL. Aldo-keto reductases AKR1C1, AKR1C2 and AKR1C3 may enhance progesterone metabolism in ovarian endometriosis. Chem Biol Interact. 2011;191:217-26.

37. Davidson B, Smith Y, Nesland JM, Kærn J, Reich R, Trope' CG. Defining a prognostic marker panel for patients with ovarian serous carcinoma effusion. Hum Pathol. 2013;44:2449-60.

\section{Submit your next manuscript to BioMed Central and take full advantage of:}

- Convenient online submission

- Thorough peer review

- No space constraints or color figure charges

- Immediate publication on acceptance

- Inclusion in PubMed, CAS, Scopus and Google Scholar

- Research which is freely available for redistribution 